INRA Prod. Anim.,

2011, 24 (3), 221-234

\title{
La sélection génétique pour la maîtrise des strongyloses gastro-intestinales des petits ruminants
}

\author{
C. DE LA CHEVROTIÈREI, C. MORENO ${ }^{2}$, P. JAQUIET ${ }^{3}$, N. MANDONNET ${ }^{1}$ \\ ${ }^{1}$ INRA, UR143, Recherches Zootechniques, Domaine Duclos, Prise d'eau, F-97170 Petit-Bourg, France \\ 2 INRA, UR631 Amélioration Génétique des Animaux, F-31326 Castanet-Tolosan, France \\ ${ }^{3}$ UMR INRA/DGER1225, Interactions Hôtes-Agents Pathogènes, École Nationale Vétérinaire, \\ 23, Chemin des Capelles, F-31076 Toulouse, France \\ Courriel : Nathalie.Mandonnet@antilles.inra.fr
}

L'omniprésence des parasites gastro-intestinaux et leur pouvoir pathogène fragilisent les systèmes d'élevage, particulièrement en milieu tropical humide. La sélection génétique est une méthode efficace sur le long terme pour maîtriser ces parasites au pâturage. Les nouveaux outils de génétique quantitative et de génomique ouvrent de nouvelles perspectives dans ce domaine.

Dans le contexte de l'élevage au pâturage, les Strongyloses GastroIntestinales (SGI) représentent une contrainte pathologique majeure des petits ruminants en zone tempérée et, particulièrement, en zone tropicale humide (Over et al 1992), du fait de pertes de production (lait, croissance, laine) et de mortalités induites. La mâ̂trise chimique du parasitisme ayant démontré ses limites (Sangster 1999), la pérennité des systèmes d'élevage basés sur le pâturage est à rechercher dans la mise en œuvre de techniques innovantes (Waller 1997). L'objectif n'est plus désormais de soustraire les animaux aux contraintes du milieu d'élevage mais de les choisir pour leur adaptation à ces contraintes.

Depuis quelques années, la maîtrise des SGI passe par la manipulation des équilibres hôtes-parasites dans les systèmes pâturés par combinaison de diverses stratégies (Mahieu et al 2009). La résistance génétique des petits ruminants aux SGI s'inscrit dans cette nouvelle démarche et y tient un rôle majeur. La mise en évidence d'une variabilité génétique de ce caractère remonte aux années 1940-1950 avec les travaux de Gregory (1937) et Emik (1949). Elle a fait ensuite l'objet de nombreux développements à partir des années 1980.

L'objectif de cette synthèse bibliographique est de souligner comment les diverses approches de la génétique aident à mieux comprendre et utiliser en sélection la résistance aux SGI des petits ruminants. Au préalable, il est nécessaire de rappeler quelques définitions car le terme de résistance peut prendre de nombreuses acceptions.

\section{1 / Qu'est ce que la résistan- ce aux strongyloses gastro- intestinales ? Comment la mesure-t-on ?}

Jusqu'à il y a 8-10 000 ans, les ruminants ont évolué en équilibre avec leurs populations parasitaires. La domestication des mammifères par l'Homme est venue rompre un équilibre naturel (Mignon-Grasteau et al 2005). En effet, les animaux ont été parqués sur des surfaces restreintes ce qui a considérablement modifié l'épidémiologie des parasitoses gastro-intestinales (Thamsborg et al 1996). Le détenteur d'animaux est entré petit à petit dans une logique d'augmentation de la production de son troupeau. Selon la théorie de l'allocation des ressources (Beilharz et al 1993), en l'absence d'apport alimentaire compensatoire, l'effort de sélection sur ces caractères de production a entraîné une réallocation des ressources alimentaires et une dépression génétique des autres caractères tels que la reproduction et l'adaptation au milieu (Menendez-Buxadera et Mandonnet, 2006). Le parasite s'est ainsi trouvé favorisé et les défenses des hôtes ont été diminuées. Toutefois, l'impact n'a pas été le même chez tous les hôtes. Il existe une variabilité individuelle dans la résistance des animaux d'un troupeau. Le parasite s'appuie sur un petit nombre d'animaux très sensibles pour réaliser rapidement son cycle (Herbert et Isham 2000), produire un grand nombre de propagules et coloniser efficacement le milieu.

Les SGI infestant les petits ruminants sont des nématodes de l'ordre des Strongylida, et de la famille des Trichostrongylidae, d'où leur nom commun de strongles. Leur cycle biologique se déroule en deux phases (figure 1) et n'implique qu'un seul hôte (monoxénie). La phase «libre ou externe» débute par l'expulsion des œufs pondus par les vers femelles dans les fèces des animaux sur le pâturage. Ensuite, deux stades larvaires (L1 et L2) successifs sont nécessaires pour aboutir à la larve infestante, dite larve L3. L'ingestion des larves infestantes par les animaux, marque le début de la «phase parasite ou phase interne». Les larves migrent alors vers la muqueuse digestive de leur site d'implantation, où se déroulent deux mues aboutissant à l'adulte mâle ou femelle. Une fois la maturité sexuelle atteinte, le ver femelle pond ses oufs dans la lumière du tube digestif qui seront excrétés à nouveau avec les matières fécales de l'hôte (Rahman et Collins, 1990).

Le terme de résistance recouvre différents phénomènes biologiques. Au sens strict, la résistance se définit comme la 
Figure 1. Cycle de développement des strongles gastro-intestinaux.

L1, L2, L3 et L4 représentent les stades larvaires successifs du développement de l'œuf jusqu'à l'âge adulte des strongles gastro-intestinaux.
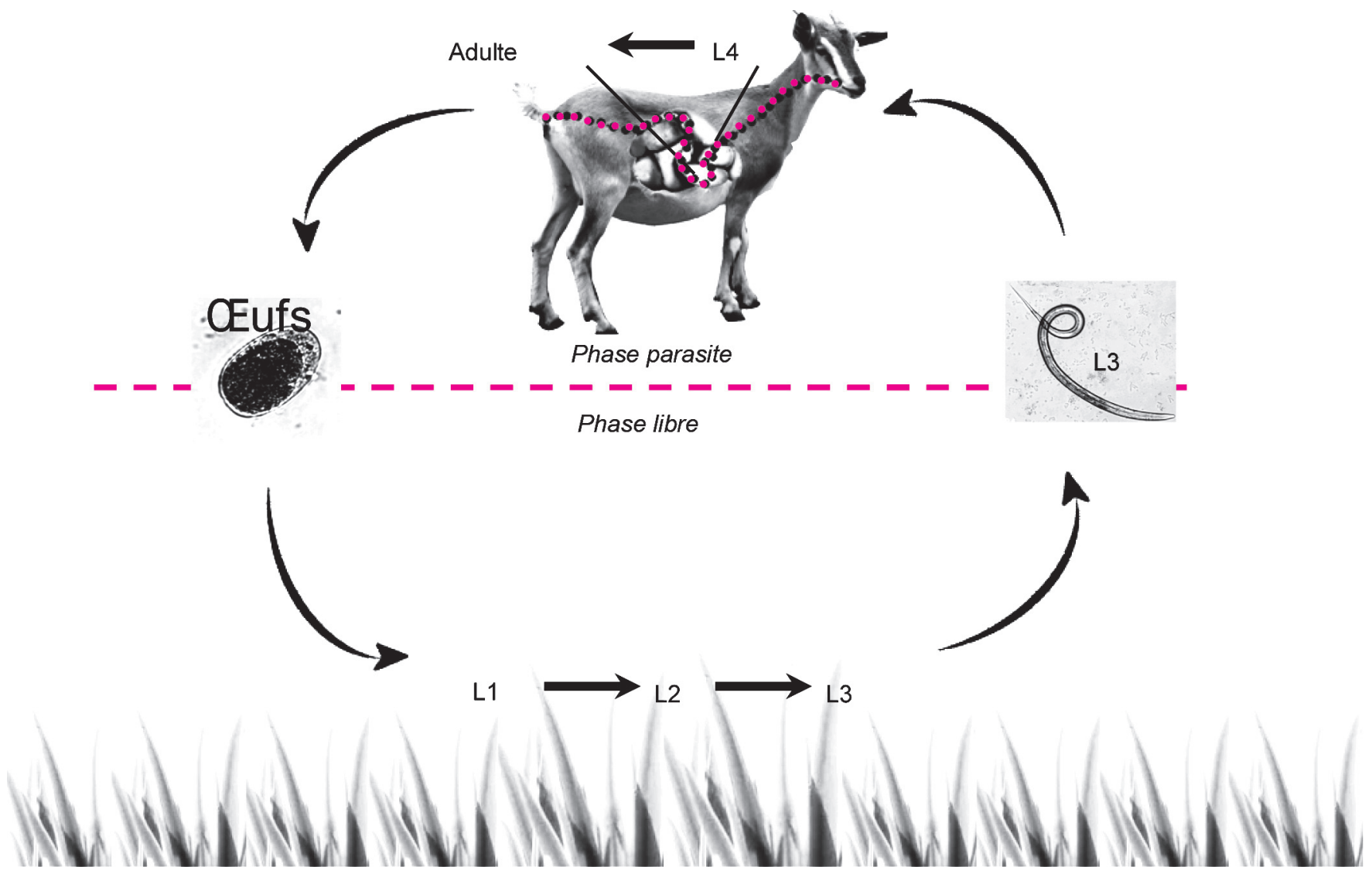

capacité d'un animal à limiter la taille de la population vermineuse hébergée, c'est-à-dire à prévenir l'installation des larves et/ou à provoquer leur élimination rapide et celles des adultes. Le nombre de vers installés est l'évaluation la plus juste et la plus directe de la résistance d'un animal, mais elle ne peut se mesurer qu'après abattage. Elle est donc difficilement envisageable dans un projet de sélection. De ce fait, le critère de sélection le plus fréquemment retenu comme indicateur phénotypique de résistance est l'excrétion d'œufs exprimée en nombre d'œufs par gramme de fèces (Baker 1997). C'est une estimation indirecte de la charge parasitaire de l'animal mais une évaluation directe de son pouvoir contaminateur, c'est-à-dire du nombre d'œufs excrétés sur le pâturage et capables d'assurer ainsi la réinfestation des animaux. La résistance/ sensibilité d'un animal au sens strict, ne préfigure pas systématiquement de sa tolérance, aptitude de l'animal à survivre aux effets pathogènes du parasitisme (Albers et al 1987), ni de sa résilience, aptitude de l'animal à maintenir sa production en niveau subclinique d'infestation. Or ces acceptions sont les plus «parlantes» en termes de productivité d'élevage.
La résistance aux SGI est un phénomène complexe par le nombre de mécanismes physiologiques impliqués. L'estimer par un seul paramètre phénotypique expliquant l'ensemble des processus inhérents est difficile (Dominik 2005). On a donc recours à un ensemble de paramètres pour caractériser un statut de résistance ou de sensibilité. Ces paramètres peuvent être classés en trois catégories: les paramètres parasitologiques, immunologiques et physiopathologiques. Les paramètres parasitologiques (excrétion d'œufs, charge parasitaire) sont relatifs à des mesures du cycle parasitaire et reflètent le niveau d'infestation de l'animal. Les paramètres immunologiques (anticorps, éosinophilie sanguine et tissulaire, mastocytes, etc.) estiment la résistance à travers l'intensité des réactions immunitaires de l'hôte en présence de nématodes. Enfin, les paramètres physiopathologiques (hématocrite, albuminémie, pepsinogène sérique, «dag score») évaluent les effets délétères de l'infestation parasitaire et reflètent la résilience des hôtes. La résilience s'évalue en perte de croissance par rapport à des animaux sains, ou encore comme une fréquence de traitements anthelminthiques indispensables pour maintenir un bon niveau de croissance (infestation par Trichostrongylus colubriformis), ou pour contenir l'intensité de l'anémie lors d'infestation par Haemonchus contortus (méthode Famacha $\odot$, Mahieu et al 2007). La tolérance s'exprime quant à elle en termes de chance de survie à l'exposition à un stress parasitaire sévère (Mandonnet et al 2003).

La composante immunitaire de la résistance est clairement impliquée. La réponse immune des ovins vis-à-vis des SGI est bien décrite (Terefe et al 2007). Leur résistance semble directement reliée à la mise en place d'une réponse immunitaire adaptative de type Th2 (figure 2). Elle induit la prolifération de mastocytes, de leucocytes et d'éosinophiles dans la muqueuse gastro-intestinale. Cette réponse produit également des immunoglobulines A, G1 et E spécifiques. L'expression de gènes impliqués dans la résistance aux SGI a été récemment étudiée chez l'espèce ovine (Diez-Tascon et al 2005, Keane et al 2007, Rowe et al 2009, MacKinnon et al 2009, Andronicos et al 2010). Ces études ont mis en évidence une expression différentielle de plusieurs gènes du Complexe Majeur d'Histocompatibilité $(\mathrm{CMH})$, ou impliqués dans la structure 
Figure 2. Représentation schématique de la balance Th1/Th2 de la réponse immunitaire aux SGI (l'orientation Th2 est la plus détaillée). CPA : Cellule Présentatrice de l'Antigène; Th: Lymphocyte $T$ auxiliaire/helper; B: Lymphocyte B; Pla: Plasmocyte ; Ma : Mastocyte ; Eo : Eosinophile. (Adaptée de Neurath et al 2002).

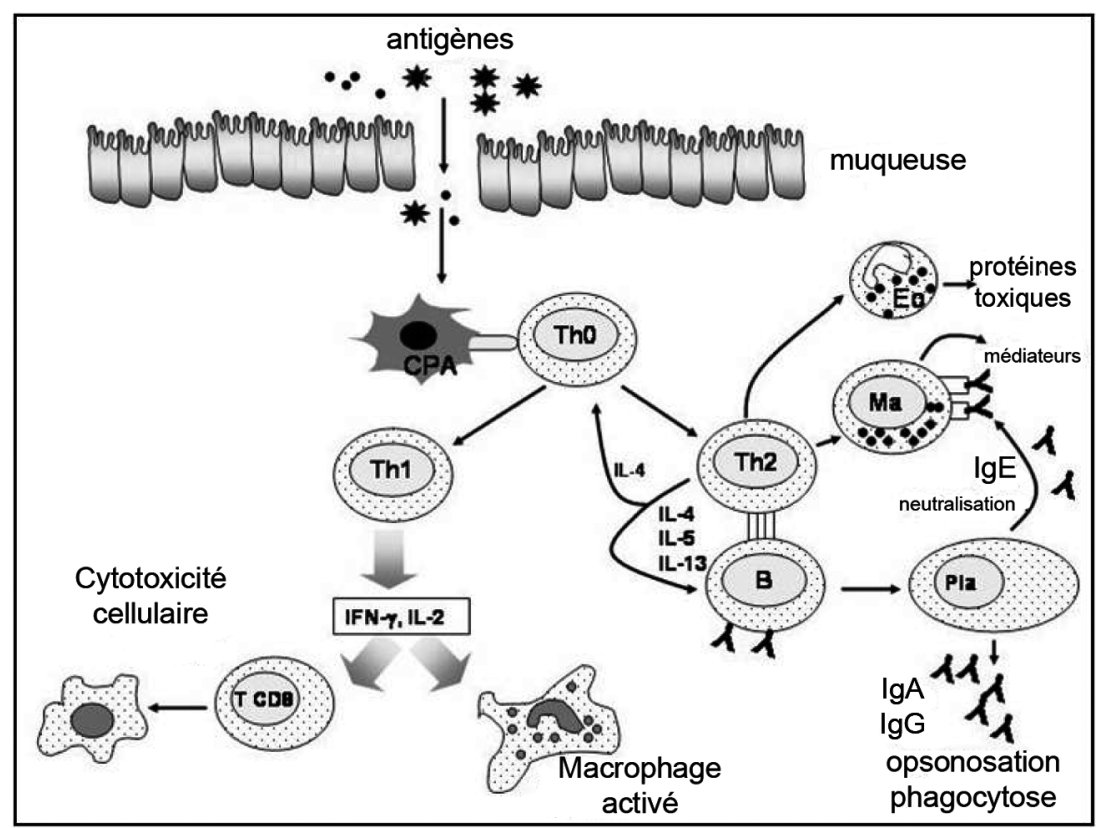

du muscle lisse intestinal chez des moutons résistants et sensibles (Diez-Tascon et al 2005, Keane et al 2007). Malgré tout, les hypothèses restent nombreuses quant à l'enchaînement des mécanismes et l'identification du mécanisme dominant, décisif dans cette chaîne. Les travaux sont rares chez les caprins. Les premières hypothèses ont été avancées en chèvre Créole (Bambou et al 2009).

Il est primordial, de comprendre suffisamment les mécanismes d'adaptation sur lesquels le sélectionneur veut faire levier et de s'assurer qu'ils ne sont pas antagonistes avec ceux régulant la production ou la résistance à d'autres maladies de l'animal.

\section{2 / Apports de la génétique quantitative à la maîtrise des strongyloses gastro- intestinales-expériences de sélection}

Les premiers travaux sur la résistance génétique aux SGI avaient pour objectif de mettre en évidence une variabilité inter- et intra-races exploitable en sélection et d'estimer les paramètres génétiques de la résistance, indicateurs de l'efficacité d'une telle sélection. Les Néo-Zélandais (Watson et al 1986) et les Australiens (Woolaston et al 1991) ont été pionniers en la matière, car confrontés intensément au problème du parasitisme. La majorité des études concerne l'espèce ovine mais quelques données existent dans l'espèce caprine, dont la chèvre Créole.

\section{1 / Exploitation des différences entre races}

Des différences de résistance entre populations de petits ruminants ont été observées dans tous les grands types de systèmes de production en zone tropicale ou tempérée, et pour une grande variété d'espèces parasites $(H$. contortus, T. colubriformis, Teladorsagia circumcincta, Oesophagostomum columbianum, Nematodirus sp.), en infestation artificielle ou naturelle (tableau 1).

Les races identifiées comme résistantes sont souvent des races locales originaires de régions humides, probablement parce qu'elles ont subi une plus forte pression de sélection naturelle par les parasites en l'absence de traitement et sont en équilibre avec leur milieu (moutons Florida Native, Gulf Coast Native, Blackbelly et Sainte Croix, chèvre Small East African et Créole). Cela leur confère une meilleure résilience (aptitude à maintenir sa production) en milieu parasité et donc une meilleure productivité (mouton Red Massaï au Kénya - Baker et al 2003; mouton Garole en Inde - Nimbkar et al 2003). Les races spécialisées plus sensibles ne parviennent pas à exprimer leur potentiel de production en présence d'une pression parasitaire sévère dans des systèmes d'élevage à faible niveau d'intrants.
Les races de moutons à poils (d'origine tropicale) semblent plus résistantes que la plupart de leur homologues à laine d'origine tempérée (MacKinnon et al 2010, Gruner et al 2004, Gonzalez et al 2008), conséquence d'une sélection naturelle en milieu fortement parasité. Les chèvres laitières sont également plus sensibles que les allaitantes, probablement parce que leurs besoins nutritionnels supérieurs à des fins de production les amènent à consommer plus d'herbe et donc plus de parasites (Hoste et al 2001).

En milieu tropical (fortement parasité), ces connaissances orientent vers l'utilisation des races locales adaptées à leur milieu, en race pure ou en croisement terminal avec des races plus productives. Suivant le milieu et le degré de spécialisation des populations de petits ruminants utilisés localement, la démarche sera sensiblement différente. En zone tempérée, la maîtrise des SGI par substitution ou croisement se heurte au niveau de productivité élevé des races exploitées et cette voie n'est pas utilisée à ce jour. A notre connaissance, aucune expérience commerciale n'a été tentée en ce sens, même en Afrique du Sud ou en Australie où l'élevage ovin peut être remis en cause par le parasitisme. Dans un futur plus ou moins proche, on pourrait envisager l'introgression de gènes (allèles) de résistance identifiés dans une race résistante de région humide vers une population tempérée spécialisée. Cette approche permettrait d'introduire des polymorphismes de résistance existant dans des races peu productives chez des races cibles sensibles au parasitisme des milieux tempérés par exemple.

Dans les systèmes d'élevage tropicaux en revanche, les expériences de substitution de races locales par des races exotiques spécialisées, ont été nombreuses mais ont invariablement échoué à court ou moyen terme. Les races tempérées ont un mauvais comportement dans les systèmes d'élevage à faible niveau d'intrant et fortes contraintes (Ayalew et al 2003). Cependant, il est possible de substituer une race locale par une race tropicale plus résistante (St Croix résistant en Indonésie et Philippines, BlackBelly résistant en Indonésie).

L'amélioration génétique par croisement ou introgression pour la résistance aux SGI paraît une stratégie séduisante mais elle est par ailleurs lourde et coûteuse à mettre en place (introduction de reproducteurs d'une nouvelle race, maintien de plusieurs noyaux de race pure en cas de croisement...). Dans l'état actuel des connaissances, le rapport bénéfice/coût n'est pas encore 
Tableau 1. Revue bibliographique des études de variabilité inter-race de la résistance aux strongles chez les différentes races ovines et caprines.

\begin{tabular}{|c|c|c|c|c|}
\hline \multicolumn{2}{|c|}{ Race résistante } & \multirow{2}{*}{$\begin{array}{l}\text { Races comparées } \\
\text { Galla }\end{array}$} & \multirow{2}{*}{\begin{tabular}{|l} 
Pays \\
Kenya
\end{tabular}} & \multirow{2}{*}{\begin{tabular}{|l|} 
Références \\
Baker et a/ 1998, 2001
\end{tabular}} \\
\hline Chèvres & Small East African & & & \\
\hline & Red Sokoto & Sahelian & Nigéria & Makun et a/ 2008 \\
\hline & Saanen & Angora & France & Hoste et a/ 2001 \\
\hline & West African Dwarf & Sahelian & Nigéria & Chiejina et a/ 2010 \\
\hline & Anglo-Nubians & Caninde & Brésil & Costa et a/ 2000 \\
\hline & Thai Native & $50 \%$ TN $\times 50 \%$ AN & Thaïlande & Pralomkarn et a/ 1997 \\
\hline & Barbari & Jamunapari & Inde & Chauhan et al 2003a,b \\
\hline \multirow[t]{22}{*}{ Moutons } & Gulf Coast Native & Suffolk & Etats-unis & $\begin{array}{l}\text { Miller et al 1998, 2006, Li et al } \\
\text { 2001, }\end{array}$ \\
\hline & St-Croix X Sumatra & Sumatra & Indonésie & Romjali et a/ 1997 \\
\hline & Java Fat-tail X Sumatra & Sumatra & Indonésie & Romjali et a/ 1997 \\
\hline & $\begin{array}{l}\text { Barbados Blackbell X } \\
\text { Sumatra }\end{array}$ & Sumatra & Indonésie & Romjali et a/ 1997 \\
\hline & Menz & Horro & Éthiopie & $\begin{array}{l}\text { Tembely et al 1998, Rege et al } \\
\text { 2002, Haile et a/ } 2002\end{array}$ \\
\hline & Red Massai & Dorper & Kenya & $\begin{array}{l}\text { Mugambi et al 1996, Wanyangu } \\
\text { et al 1997, Baker et al } 2003\end{array}$ \\
\hline & Red Massai & $\begin{array}{l}\text { Dorper, Romney Marsh, } \\
\text { Blackheaded Somali }\end{array}$ & Kenya & Mugambi et a/ 1997 \\
\hline & Crioula Lanada & Corriedale & Brésil & Bricarello et a/ 2004 \\
\hline & Lohi & Kachhi, Thalli & Pakistan & Saddiqi et a/ $2010 a, b$ \\
\hline & Criollo & Suffolk & Mexique & Alba-Hurtado et a/ 2010 \\
\hline & $\begin{array}{l}\text { Santa Ines pure and } \\
\text { crossbred }\end{array}$ & Suffol, lle de France & Brésil & Amarante et a/ 2004, 2009 \\
\hline & Local Kashmiri & $\begin{array}{l}\text { Crossbred Kashmir Merino } \\
\text { > Bhakarwal, Corriedale }\end{array}$ & Inde & Tariq et a/ 2008 \\
\hline & St-Croix hair & $\begin{array}{l}\text { Wool (50\%Dorset, } \\
\text { 25\%Rambouillet, } 25 \% \\
\text { Finnsheep) }\end{array}$ & États-Unis & MacKinnon et a/ 2010 \\
\hline & Canaria Hair & Canaria & Espagne & Gonzalez et a/ 2008 \\
\hline & Garole sheep & Decanni, Bannur & Inde & Nimbkar et al 2002, 2003 \\
\hline & Blackbelly & INRA401 & Guadeloupe & $\begin{array}{l}\text { Aumont et a/ 2003, Gruner et al } \\
2003\end{array}$ \\
\hline & Djallonké & Djallongé-Sahelian & Gambie & Goossens et al 1999 \\
\hline & Merinoland & Rhoen & Allemagne & $\begin{array}{l}\text { Gauly et al 2002, Hielscher et al } \\
2006\end{array}$ \\
\hline & $\begin{array}{l}\text { Dorper, Katahdin, St- } \\
\text { Croix (Hair) }\end{array}$ & Hampshire & Etats-Unis & Burke et Miller, 2002, 2004 \\
\hline & Florida Native, FN x R & Rambouillet & Etats-Unis & Amarante et a/ 1999 \\
\hline & Texel & Suffolk & Irlande & Good et a/ 2006 \\
\hline & Sabi & Dorper & Zimbabwe & Matika et al 2003 \\
\hline
\end{tabular}


assez clair que ce soit pour l'amélioration de la productivité en zone tempérée, ou pour justifier l'investissement dans des infrastructures adaptées en zone tropicale (Alexandre et Mandonnet 2005).

En 2008, les éleveurs caprins de Guadeloupe ont opté pour l'exploitation de leur population locale, la chèvre Créole, issue du métissage de races africaines et européennes durant la colonisation. Ils la destinent à la production de chevreaux de race pure et de chevreaux terminaux Boer $\mathrm{x}$ Créole pour la boucherie. Pour ce faire, ils exploiteront la variabilité intra-race Créole, autre voie d'amélioration génétique de la résistance aux SGI

\section{2 / Exploitation de la variabili- té intra-race}

La majorité des travaux conduits intra-race se sont attachés à quantifier la variabilité génétique disponible intra populations ovines en vue d'inclure ce caractère dans les schémas de sélection. Chez les ovins, les résultats montrent une héritabilité moyenne (comprise entre 0,2 et 0,4 en général) de la résistance, mesurée classiquement par l'excrétion d'œufs, quel que soit la race de l'hôte (tableau 2). Une sélection efficace est donc envisageable. Dans le cas des caprins, les études sont peu nombreuses et parfois contradictoires. Certaines concluent à l'absence de variabilité génétique (Woolaston et al 1992) et

Tableau 2. Héritabilités du caractère de résistance aux SGI pour des populations ovines et caprines (en gras).

\begin{tabular}{|c|c|c|c|c|c|}
\hline Région & $\begin{array}{c}\text { Type } \\
\text { infestation }\end{array}$ & $\begin{array}{c}\text { Espèces } \\
\text { nématodes }\end{array}$ & Race & Héritabilité & Références \\
\hline Tropical & IN & Mixte & Fijian & $0,04 \pm 0,03$ & Woolaston et al 1992 \\
\hline \multirow[t]{8}{*}{ Subtropical } & IN & Mixte & $\begin{array}{l}\text { Galla + Small East } \\
\text { African }\end{array}$ & $0,20 \pm 0,14$ & Baker et a/ 2001 \\
\hline & IN & Mixte & Barbari & $0,13 \pm 0,04$ & $\begin{array}{l}\text { Mandal et Sharma, } \\
2008\end{array}$ \\
\hline & IN & Mixte & Creole & $0,33 \pm 0,06$ & Mandonnet et a/ 2001 \\
\hline & IN & Mixte & Avikalin & $0,15 \pm 0,10$ & Prince et al 2010 \\
\hline & IN & Mixte & Red Massai & $0,33 \pm 0,10$ & Baker et al 1994 \\
\hline & IN & Mixte & Dorper & $0,31 \pm 0,14$ & Baker et a/ 2003 \\
\hline & IN & Mixte & Santa Ines & $0,18 \pm 0,05$ & McManus et a/ 2009 \\
\hline & IE & $\mathrm{Hc}$ & Garole et Decanni & $0,36 \pm 0,22$ & Nimbkar et a/ 2003 \\
\hline \multirow[t]{24}{*}{ Tempéré } & IE -IN & Tc-Mixte & Australian Angora & $0,12 \pm 0,08$ & Bolormaa et a/ 2010a \\
\hline & IN & Mixte & Cross bred cashmere & $0,17 \pm 0,02$ & Vagenas et a/ 2002 \\
\hline & IN & Mixte & Saanen & $0,05 \pm 0,03$ & Morris et a/ 1997b \\
\hline & IN & Mixte & $\begin{array}{l}\text { Blueface Leicaster X } \\
\text { Scottish Blackface }\end{array}$ & $0,09 \pm 0,04$ & Wolf et a/ 2008 \\
\hline & IE & $\mathrm{Hc}, \mathrm{Tc}$ & INRA401 & 0,39 à 0,48 & Gruner et a/ 2004 \\
\hline & IN & Mixte & Rhön & $0,44 \pm 0,11$ & Gauly et Erhardt, 2001 \\
\hline & IN & Mixte & Polish longwool & 0,20 à 0,33 & Bouix et a/ 1998 \\
\hline & IE & $\mathrm{Hc}$ & $\begin{array}{l}50 \% \text { Dorset, } 25 \% \\
\text { Romney } 25 \% \text { Finnsheep }\end{array}$ & 0,19 & Vanimisetti et al 2004 \\
\hline & IN & Mixte & Perendale & $0,22 \pm 0,03$ & Morris et a/ 2005 \\
\hline & IN & Mixte & Texel & 0,17 à 0,25 & Bishop et a/ 2004 \\
\hline & IN & Mixte & Soay & $0,26 \pm 0,01$ & Beraldi et a/ 2007 \\
\hline & IN & Mixte & Scottish Blackface & $0,33 \pm 0,15$ & Davies et a/ 2005 \\
\hline & IN & Mixte & Menz & $0,01 \pm 0,02$ & Rege et a/ 2002 \\
\hline & IN & Mixte & Crossbred romney & $0,34 \pm 0,20$ & Watson et a/ 1986 \\
\hline & IN & Mixte & Romney & $0,42 \pm 0,06$ & Morris et al 1997a \\
\hline & IN & Mixte & Romney & $0,13 \pm 0,07$ & McEwan et a/ 1992 \\
\hline & IN & Mixte & Romney & $0,35 \pm 0,02$ & Morris et a/ 2004 \\
\hline & IE & $\mathrm{Hc}$ & Merinoland & $0,17 \pm 0,07$ & Gauly et a/ 2002 \\
\hline & IE & $\mathrm{Hc}$ & Merino-HR-LR & $0,49 \pm 0,17$ & Sréter et a/ 1994 \\
\hline & IE & $\mathrm{Hc}$ & Merino & $0,29 \pm 0,03$ & $\begin{array}{l}\text { Woolaston et Piper, } \\
1996\end{array}$ \\
\hline & IE & $\mathrm{Hc}$ & Merino & $0,26 \pm 0,09$ & Albers et al 1987 \\
\hline & IN & Mixte & Merino & $0,21 \pm 0,02$ & Khusro et a/ 2004 \\
\hline & IE & Tc & Merino & $0,39 \pm 0,05$ & $\begin{array}{l}\text { Woolaston et Windon, } \\
2001\end{array}$ \\
\hline & IN & Mixte & Merino & $0,33 \pm 0,11$ & Pollot et a/ 2004 \\
\hline
\end{tabular}

1 IN : Infestation Naturelle; IE : Infestation Expérimentale.

2 HC : Haemonchus Contortus ; TC : Trichostrongylus Colubriformis. 
d'autres à la possibilité de sélectionner efficacement (Baker et al 2001). L’hétérogénéité des estimations est certainement liée à la plus ou moins bonne maîtrise de la variabilité environnementale des dispositifs, ou à la fixation d'allèles dans les populations résistantes. Dans ce cas, la variabilité génétique disponible pour la sélection en caprin paraît moitié moins importante que chez les ovins avec une héritabilité voisine de 0,2 (tableau 2). Ceci peut être dû au fait que les mécanismes de résistance seraient moins efficaces chez la chèvre que chez le mouton. En effet, les chèvres ont une alimentation principalement de type «cueilleur» tandis que les ovins, de type «brouteur» (Hofmann 1989), sont soumis à une pression de sélection plus importante de la part des parasites gastrointestinaux car en contact continu avec les larves infestantes sur l'herbe. La coévolution plus intime entre les ovins et les strongles aurait ainsi abouti à des mécanismes de résistance plus complexes, impliquant un plus grand nombre de gènes et initiant ainsi une plus grande variabilité génétique (Mirkena et al 2010), ce qui pourrait avoir généré une variabilité génétique de ce caractère plus importante que chez les chèvres en impliquant un plus grand nombre de gènes. Par ailleurs, quelque soit l'espèce, une fois le sevrage passé, le caractère de résistance n'est plus contrôlé par des caractères maternels (Baker et al 1998, Bishop et Stear 2001). Ce sont uniquement les gènes de résistance de l'individu qui s'expriment. Cette absence d'effets génétiques maternels après sevrage simplifie la sélection et augmente son intérêt.

Chez les ovins, une fois l'immunité de l'hôte acquise vis-à-vis des strongles, les mécanismes permettant la résistance en limitant l'installation et le développement de la population vermineuse, sont efficaces quelque soit le mode d'infestation expérimental ou naturel (Gruner et al 2004, Watson et al 1992, Woolaston et Eady 1995). Ce résultat permet d'envisager de sélectionner des reproducteurs sur leur résistance à une infestation expérimentale pour maîtriser le parasitisme de leurs descendants. De plus, des études chez les ovins ont pu montrer l'absence de spécificité de la résistance vis-àvis de la souche de parasite et de l'espèce de parasite (Gruner et al 2004). Notamment, un animal sélectionné pour sa résistance à $H$. contortus présentera un certain niveau de protection vis-à-vis de T. colubriformis, les espèces dominantes en zone tropicale. En revanche, peu d'études (Carta et al 2009) ont abordé un éventuel antagonisme entre la résistance aux SGI et la résistance à des pathogènes d'une autre nature (virus, bactéries...). Enfin, les mécanismes génétiques impliqués dans les phénomènes de résistance sont pour partie identiques aux différents stades de production (Woolaston et al 1992, Morris et al 1998, Mandonnet et al 2006). Ainsi, la sélection d'animaux résistants en phase de croissance diminue également la sensibilité des chèvres autour de la mise bas, période pendant laquelle une levée de l'immunité des mères favorise ensuite l'infestation des jeunes sous la mère.

Par ailleurs, la grande majorité des études concluent à des relations favorables ou neutres entre résistance et caractères de production ou de reproduction. Vanimissetti et al (2004) ont montré l'absence de contrôle génétique commun entre caractères de reproduction et résistance aux SGI chez l'agneau. Dans leur revue bibliographique, Safari et al (2005) rapportent une corrélation génétique moyenne de - 0,24 entre le poids et l'excrétion d'œufs chez les ovins. Chez la chèvre Créole, des relations neutres ont été estimées entre fertilité, prolificité, valeur laitière et excrétion d'œufs tandis que la corrélation avec le poids à la mise en reproduction est légèrement favorable (Gunia et al 2011). En revanche, la relation entre la résistance et la croissance en milieu non infesté n'est pas connue. Les gènes qui contrôlent la résistance semblent donc peu (ou pas) impliqués dans le déterminisme des caractères productifs. L'amélioration génétique de la résistance des petits ruminants ne va donc pas a priori à l'encontre de l'amélioration de ces caractères. Cependant, les corrélations phénotypiques défavorables observées dans certaines études traduisent le coût métabolique de la réponse immunitaire contre les SGI (Colditz 2002).

Les critères classiques de mesure de la résistance sont l'excrétion d'œufs et l'hématocrite. Ces critères sont très corrélés bien que ne recouvrant pas les mêmes aspects du caractère de résistance. En effet, l'hématocrite corrélé fortement avec les variables de poids, est plus considéré comme un critère de résilience quelle que soit l'espèce parasite dominante (Gunia et al 2011). La résilience peut être également prise en compte dans un schéma en concentrant l'effort de sélection sur les caractères de production dans le milieu contraignant et en laissant la composante adaptation sous l'effet de la sélection naturelle. Cette voie est moins efficace qu'une prise en compte directe de la résistance, en particulier parce que les antagonismes génétiques entre production et adaptation sont négligés (Finch 1986, Davis 1993). Cependant, du progrès génétique peut être obtenu (Poivey
1987). Par ailleurs, la sélection doit intervenir dans un milieu le plus proche possible du milieu de production afin d'éviter les interactions génotype $x$ environnement très fréquentes en milieu contraignant (Vercoe et Frish 1992, Menendez-Buxadera et Mandonnet 2006).

Dans le cas de la résistance aux SGI, beaucoup de connaissances sont déjà disponibles (Doeschl-Wilson et al 2008) et le sélectionneur désirant l'inclure directement dans son schéma d'amélioration génétique se trouve dans une situation favorable. D'après les relations génétiques connues en milieu très infesté, la prise en compte de la résistance aux SGI dans le schéma de sélection ne devrait pas détériorer la sélection sur les autres caractères. La situation est moins claire en cas d'infestations faibles ou saisonnières et nécessite des études complémentaires sur les relations génétiques entre résistance et production lorsque le milieu est faiblement infesté (climat continental) (Doeschl-Wilson et al 2008).

Enfin, contrairement aux caractères de production, la sélection sur la résistance à une maladie peut conduire à l'apparition de nouveaux pathogènes adaptés aux animaux résistants. Ce type de problème a été observé lors de sélections ciblées sur un seul gène dans d'autres maladies, mais ce risque est bien moindre lorsque la sélection concerne un caractère polygénique ou plusieurs gènes connus (Saulai et al 2001, Kemper et al 2009).

En pratique, des programmes de sélection se sont mis en place dans certains pays en utilisant comme critère l'intensité d'excrétion d'œufs dans les matières fécales, mesure qui reflète le nombre de vers installés, leur développement dans le tractus digestif et la prolificité des vers femelles. En Australie, tous ces résultats ont permis la mise en place d'un service nommé WormBoss, développé par l'Australian Sheep Industry Cooperative Research Centre (Sheep CRC) et l'Australian Wool Innovation (AWI), pour aider les éleveurs à contrôler les populations vermineuses dans leurs élevages et à sélectionner les béliers résistants aux SGI. Au Kenya, Gicheha et al (2005, 2007) ont proposé un objectif de sélection pour les élevages d'ovins producteurs de viande les plus productifs en zone tropicale, incluant la résistance aux SGI. En Guadeloupe, un schéma de sélection des caprins Créole combinant résistance aux SGI, qualités maternelles et production de viande dans un objectif économique synthétique, est en cours de mise en œuvre (Gunia et al 2010). 


\section{3 / Peut-on envisager l'utili- sation d'informations géno- miques pour améliorer la résistance génétique aux strongyloses gastro-intesti- nales?}

L'hypothèse est faite qu'il sera possible d'améliorer la sélection pour la résistance aux SGI en utilisant des marqueurs moléculaires permettant de détecter les polymorphismes de l'ADN responsables de la variabilité génétique globale estimée ou utilisée précédemment.

\section{1 / La recherche de QTL de résistance aux SGI chez les petits ruminants}

Dans les années 1990, l'essor des technologies de biologie moléculaire a fait naître de grands espoirs chez les sélectionneurs. L'information moléculaire est venue compléter l'information phénotypique et généalogique pour tenter d'identifier les gènes expliquant les phénomènes de résistance. Mais à cause du nombre limité de marqueurs alors disponibles, la localisation des gènes était très imprécise et leur présence incertaine. Dans un premier temps, ces études n'ont donc pas permis de modifications profondes des stratégies de sélection.

De nombreux programmes de recherche à travers le monde ont donc concerné la détection de Quantitative Trait Loci (QTL) de résistance aux SGI chez les petits ruminants. Deux stratégies ont été utilisées. La première, nommée «approche par gène candidat», étudie la relation entre un caractère et un gène connu qui serait associé aux mécanismes physiologiques impliqués dans l'expression du caractère (Sourdioux et al 1997). La seconde approche, «genome scan», consiste à étudier la relation entre un caractère et différents marqueurs génétiques répartis sur tout le génome de l'animal, sans a priori (Sourdioux et al 1997). Cette approche permet d'identifier une position possible d'un QTL sur un chromosome donné. La première stratégie peut s'avérer très efficace s'il existe une bonne connaissance préalable des mécanismes qui expliquent la variation du caractère, et donc un choix préalable d'un gène candidat. À l'inverse, la méthode du «genome scan» est une première étape et doit être poursuivie par une cartographie plus fine des régions identifiées dans les premières analyses. Le choix de la stratégie repose essentiellement sur l'état des connaissances des mécanismes entourant le caractère et le bud- get disponible. Dans le but d'identifier avec précision un gène responsable du niveau d'un caractère, les deux approches peuvent être utilisées de façon complémentaire, l'approche par «genome scan» fournissant un point d'ancrage pour une cartographie physique et l'approche par gène candidat, des arguments fonctionnels et métaboliques (Sourdioux et al 1997).

La majorité des résultats publiés concerne l'espèce ovine (Bishop et Morris 2007, Crawford et al 2006). A ce jour, seules deux études de recherche de QTL existent chez l'espèce caprine. La première, réalisée sur des chèvres australiennes, utilise l'approche par gène candidat tandis que la seconde étude est actuellement menée en guadeloupe sur la chèvre Créole en utilisant une approche de «genome scan». L'ensemble des résultats de QTL de résistance aux SGI (tableau 3), met notamment en évidence 5 régions chromosomiques principales où sont localisés des gènes impliqués dans la réponse immunitaire et qui auraient un rôle possible dans la mise en place d'une résistance aux SGI.

La région chromosomique près de l'interféron gamma (IFN- $\gamma$ ), située sur le chromosome 3 ovin, est également associée à de nombreux QTL. L'IFN- $\gamma$ est une cytokine sécrétée par les lymphocytes T CD4 + Th1. Son rôle est d'activer les macrophages et de favoriser la réponse immunitaire cellulaire de type Th1, elle induit une diminution de la polarisation $\mathrm{Th} 2$ des $\mathrm{Th} 0$. Les résultats obtenus par Coltman et al (2001) démontrent une association avec un QTL situé près de la région de l'IFN- $\gamma$ et une excrétion d'œufs faible chez le mouton de Soay. Ce QTL est aussi associé à une augmentation du niveau d'IgA chez des agneaux de 4 mois d'âge. Ces résultats suggèrent que l'effet du gène interféron sur l'excrétion d'œufs serait médié par le niveau d'IgA. En effet, une faible production de la cytokine IFN- $\gamma$ inhiberait la réponse Th1 en faveur de la réponse $\mathrm{Th} 2$ et donc augmenterait la production d'IgA.

Le chromosome 5 ovin, porteur de gènes codant pour les interleukines IL-4 et 5, a également été associé à un QTL de résistance situé près de ces gènes (Benavides et al 2002). Leur expression est capitale dans l'acquisition de la résistance aux SGI car elles polarisent la réponse immune adaptative dans le sens Th2. Les interleukines 4 et 5 sont impliquées directement dans la réponse immunitaire de type Th2, en activant la différenciation des lymphocytes $\mathrm{B}$ en plasmocytes sécréteurs d'IgA, IgE et IgG.

Le chromosome 6 ovin est associé à un QTL de résistance pour une excré- tion d'œufs faible près de gènes intervenant dans certaines réactions immunologiques. Les gènes GRO1, KIT, PDGFRA et IF1 sont localisés près du QTL trouvé. GRO1 est un oncogène impliqué dans la chimiotaxie des neutrophiles, des macrophages et des lymphocytes.

Le chromosome 18 ovin est porteur du gène codant pour la chaîne lourde $\varepsilon$ (CE) des immunoglobulines $\mathrm{E}$ (IgE) pour lequel une association entre un allèle de ce gène et la résistance à $T$. colubriformis mesurée par l'excrétion d'œufs a été mise en évidence par Clarke et al (2001). Le gène $\mathrm{C} \varepsilon$ est situé sur le chromosome $18 \mathrm{chez}$ le mouton. L'IgE est également un acteur important dans la réponse immunitaire de type Th2 et serait nécessaire à l'expulsion des vers adultes. Des niveaux élevés d'IgE ont souvent été observés chez des animaux résistants.

Le Complexe Majeur d'Histocompatibilité $(\mathrm{CMH})$, situé sur le chromosome 20 ovin, a été aussi associé avec de nombreux QTL. Des QTL situés près de cette région ont également été mis en évidence chez la chèvre sur le chromosome homologue 23 (Boloorma et al 2010b). Le CMH est une région multi-génique qui fait l'interface entre le système immunitaire et les maladies infectieuses. Les gènes codent pour des protéines réceptrices situés sur la surface de cellules immunes ou non. Le rôle principal de ces récepteurs est de reconnaître les antigènes et de les présenter aux lymphocytes $\mathrm{T}$. Les gènes du $\mathrm{CMH}$ sont divisés en deux classes distinctes : les gènes de classe I et II. Chez les mammifères, ces deux classes forment un unique complexe génique. Les molécules de classe I sont présentes dans toutes les cellules nucléées et ont essentiellement pour rôle de reconnaître des antigènes endogènes. Elles ont un rôle essentiel dans la défense contre les pathogènes intracellulaires. Les molécules de classe II sont présentes seulement sur les Cellules Présentatrices d'Antigènes (CPA) du système immunitaire. Elles reconnaissent principalement les antigènes exogènes et les présentent aux lymphocytes $\mathrm{T}$. Le système immunitaire des animaux résistants aux nématodes gastro-intestinaux aurait une plus grande capacité de reconnaissance des antigènes spécifiques des parasites, grâce aux molécules du CMH.

Cependant, de nombreuses autres régions interviennent et les QTL détectés semblent dispersés sur tout le génome suggérant, en l'état actuel des connaissances, que la résistance a fondamentalement un déterminisme polygénique (Bishop 2010). On peut remarquer, que d'un dispositif à l'autre, les 
Tableau 3. Les QTL de résistance aux strongles gastro-intestinaux mis en évidence chez les petits ruminants.

\begin{tabular}{|c|c|c|c|c|c|}
\hline Chr. ${ }^{1}$ & Population & Strongles & Caractère $^{3}$ & $\begin{array}{c}\text { Gène } \\
\text { candidat }\end{array}$ & Références $^{2}$ \\
\hline 1 & $\begin{array}{l}\text { Merinos, Romneys, } \\
\text { Spanish Churra, }\end{array}$ & $\begin{array}{l}\text { Multi-espèces, } T \text {. } \\
\text { colubriformis, } H \text {. contortus }\end{array}$ & $\begin{array}{l}\text { OPG, vers } \\
\text { adultes, IgA }\end{array}$ & & $\begin{array}{l}\text { Beh et al 2002, Diez-Tascon et al } \\
\text { 2002, Davies et al 2006, Gutiérrez-Gil } \\
\text { et al 2009, Marshall et al } 2009\end{array}$ \\
\hline 2 & $\begin{array}{l}\text { Scottish Blackface, } \\
\text { Sarde x Lacaune, } \\
\text { Merinos, Romneys }\end{array}$ & Multi-espèces, $H$. contortus & $\begin{array}{l}\text { OPG, vers } \\
\text { adultes }\end{array}$ & & $\begin{array}{l}\text { Moreno et al 2006, Crawford et al } \\
\text { 2006, Marshall et al } 2009\end{array}$ \\
\hline 3 & $\begin{array}{l}\text { Scottish Blackface, } \\
\text { Merinos, Soay, Sarde x } \\
\text { Lacaune, Blackbelly x } \\
\text { INRA401, Texel, }\end{array}$ & $\begin{array}{l}\text { Multi-espèces, } T \text {. } \\
\text { colubriformis, } H \text {. contortus, } \\
\text { T. circumcincta }\end{array}$ & OPG, IgA & IFNG & $\begin{array}{l}\text { Coltman et al 2001, Beh et al 2002, } \\
\text { Sayers et al 2005b Davies et al 2006, } \\
\text { Moreno et al 2006, Marshall et al } 2009\end{array}$ \\
\hline 4 & $\begin{array}{l}\text { Sarde } x \text { Lacaune, } \\
\text { Merinos }\end{array}$ & Multi-espèces, $H$. contortus & OPG & & Moreno et al 2006, Marshall et al 2009 \\
\hline 5 & BlackBelly x INRA401 & H. contortus & OPG, PCV & & Moreno et al 2006 \\
\hline 5 & $\begin{array}{l}\text { BlackBelly x INRA401, } \\
\text { Corriedale, Polwart } \\
\end{array}$ & Multi-espèces, $H$. contortus & OPG, PCV & $\begin{array}{l}\text { IL-3, IL- } \\
4, \text { IL-5 }\end{array}$ & $\begin{array}{l}\text { Benavides et al 2002, Moreno et al } \\
2006\end{array}$ \\
\hline 6 & $\begin{array}{l}\text { Merinos, Sarde x } \\
\text { Lacaune, Spanish } \\
\text { Churra, } \\
\end{array}$ & $\begin{array}{l}\text { Multi-espèces, } T \text {. } \\
\text { colubriformis, } H \text {. contortus }\end{array}$ & OPG & $\begin{array}{l}\text { GR01, } \\
\text { KIT, IF1 }\end{array}$ & $\begin{array}{l}\text { Beh et al 2002, Moreno et al 2006, } \\
\text { Gutiérrez-Gil et al 2009, Marshall et al } \\
2009\end{array}$ \\
\hline 7 & $\begin{array}{l}\text { BlackBelly x INRA401, } \\
\text { Merinos }\end{array}$ & H. contortus & OPG & & Moreno et al 2006, Marshall et al 2009 \\
\hline 8 & $\begin{array}{l}\text { Sarde } x \text { Lacaune, } \\
\text { Merinos, Romneys }\end{array}$ & Multi-espèces, $H$. contortus & $\begin{array}{l}\text { OPG, vers } \\
\text { adultes }\end{array}$ & & $\begin{array}{l}\text { Moreno et al 2006, Crawford et al } \\
\text { 2006, Marshall et al } 2009\end{array}$ \\
\hline 9 & Merinos & H. contortus & OPG & & Marshall et al 2009 \\
\hline 10 & $\begin{array}{l}\text { Sarde } x \text { Lacaune, } \\
\text { Spanish Churra, } \\
\text { Merinos }\end{array}$ & Multi-espèces, $H$. contortus & OPG & & $\begin{array}{l}\text { Moreno et al 2006, Gutiérrez-Gil et al } \\
\text { 2009, Marshall et al } 2009\end{array}$ \\
\hline 11 & Merinos, Romneys & $\begin{array}{l}\text { Multi-espèces, } T \text {. } \\
\text { colubriformis, } H \text {. contortus }\end{array}$ & $\begin{array}{l}\text { OPG, vers } \\
\text { adultes }\end{array}$ & & $\begin{array}{l}\text { Beh et al 2002, Crawford et al 2006, } \\
\text { Marshall et al } 2009\end{array}$ \\
\hline 12 & $\begin{array}{l}\text { Merinos, Sarde } x \\
\text { Lacaune, BlackBelly x } \\
\text { INRA401 } \\
\end{array}$ & $\begin{array}{l}\text { Multi-espèces, } T \text {. } \\
\text { colubriformis, } H \text {. contortus }\end{array}$ & OPG & & $\begin{array}{l}\text { Beh et al 2002, Moreno et al } 2006 \text {, } \\
\text { Marshall et al } 2009\end{array}$ \\
\hline 13 & \begin{tabular}{|l|} 
Sarde $\times$ Lacaune, \\
BlackBelly $x$ INRA401 \\
\end{tabular} & Multi-espèces, $H$. contortus & OPG, PCV & & Moreno et al 2006 \\
\hline 14 & $\begin{array}{l}\text { Scottish Blackface, } \\
\text { Sarde x Lacaune, } \\
\text { Spanish Churra }\end{array}$ & Multi-espèces & OPG & & $\begin{array}{l}\text { Davies et al 2006, Moreno et al 2006, } \\
\text { Gutiérrez-Gil et al } 2009\end{array}$ \\
\hline 15 & Merinos & H. contortus & OPG & & Marshall et al 2009 \\
\hline 16 & $\begin{array}{l}\text { Sarde } x \text { Lacaune, } \\
\text { Merinos }\end{array}$ & Multi-espèces, $H$. contortus & OPG & & Moreno et al 2006, Marshall et al 2009 \\
\hline 18 & Merinos & $\begin{array}{l}\text { T. colubriformis, } H \text {. } \\
\text { contortus }\end{array}$ & OPG & $\lg E$ & Clarke et al 2001, Marshall et al 2009 \\
\hline 19 & Sarde $x$ Lacaune & Multi-espèces & OPG & & Moreno et al 2006 \\
\hline 20 & $\begin{array}{l}\text { Scottish Blackface, } \\
\text { Rhönschaf, Polish } \\
\text { Heath, Soay, Merinos, } \\
\text { Suffolk, Chèvres Angora } \\
\text { et Cashmere }\end{array}$ & $\begin{array}{l}\text { Multi-espèces, } H \text {. contortus, } \\
\text { T. circumcincta }\end{array}$ & $\begin{array}{l}\text { OPG, PCV, } \\
\lg A, \lg L \\
\text { eosinophiles }\end{array}$ & $\mathrm{CMH}$ & $\begin{array}{l}\text { Schwaiger et al 1995, Buitkamp et al } \\
\text { 1996, Paterson et al 1998, Charon et al } \\
\text { 2002, Janssen et al 2002, Sayers et al } \\
\text { 2005a Davies et al 2006, Marshall et al } \\
\text { 2009, Bolormaa et al 2010b }\end{array}$ \\
\hline 21 & $\begin{array}{l}\text { Sarde } x \text { Lacaune, } \\
\text { Merinos }\end{array}$ & Multi-espèces, $H$. contortus & OPG & & Moreno et al 2006, Marshall et al 2009 \\
\hline 22 & Merinos & H. contortus & OPG & & Marshall et al 2009 \\
\hline 23 & $\begin{array}{l}\text { BlackBelly x INRA401, } \\
\text { Romneys }\end{array}$ & Multi-espèces, $H$. contortus & $\begin{array}{l}\text { OPG, } \lg G \\
\lg E\end{array}$ & & Moreno et al 2006, Crawford et al 2006 \\
\hline 24 & Merinos & H. contortus & OPG & & Marshall et al 2009 \\
\hline 25 & Merinos & H. contortus & OPG & & Marshall et al 2009 \\
\hline 26 & Merinos & H. contortus & OPG & & Marshall et al 2009 \\
\hline $\mathrm{X}$ & Merinos & H. contortus & OPG & & Marshall et al 2009 \\
\hline
\end{tabular}

1 Chromosomes ovins.

2 La localisation des QTL peut varier d'une étude à l'autre.

3 OPG : CEufs Par Gramme ; PCV : mesure d'hématocrite ; Ig : ImmunoGlobuline. 
régions QTL identifiées sont rarement identiques. On peut donc se demander si les mécanismes de résistance mis en place ne sont pas différents selon les races.

La sélection assistée par marqueurs ou par gènes n'est pas possible à l'heure actuelle pour le caractère de résistance aux SGI et les mutations causales menant à la compréhension des polymorphismes ne sont pas identifiées. Une cartographie plus fine de ces régions est nécessaire pour isoler le ou les gènes responsables de la variation génétique. La disponibilité en puces génomiques à haute densité en marqueurs et des nouvelles technologies de séquençage haut débit et à bas coût (telle que la technologie HiSeq d'Illumina) permettent d'envisager des avancées dans ce domaine chez les ovins et les caprins.

\section{2 / Les perspectives ouvertes par les puces génomiques à haute densité}

Depuis 2009, le travail effectué par le Consortium International de Génomique Ovine (ISGC, http://www.sheephapmap.org/) a permis le développement d'une puce génomique comprenant 54241 marqueurs de type Single Nucleotide Polymorphisms (SNP). La construction d'une puce comparable chez les caprins est en cours de réalisation. La révolution apportée par ces puces SNP est double. En offrant une information en tout point du génome, elles ouvrent la voie à la localisation fine et l'identification des gènes impliqués dans le déterminisme des caractères d'intérêt, comme la résistance aux SGI. En sélection, elles permettent d'accéder à un nombre de polymorphismes de l'ADN suffisant pour estimer l'effet de l'ensemble des gènes de l'animal. Ainsi, le développement des puces SNP très denses en marqueurs permet de concevoir les approches par gène candidat et par génome scan en une seule étape. Toutefois, pour la résistance aux SGI, aucun résultat n'est encore disponible avec ces nouvelles méthodes.

Dans un futur assez proche, les puces SNP permettront d'envisager une autre stratégie de sélection sur des caractères complexes, tels que la résistance aux SGI : la sélection génomique. Celle-ci repose au sens strict sur l'association entre des marqueurs génétiques et une performance. Au sein d'une population dite «de référence», des animaux sont génotypés et phénotypés, permettant ainsi de prévoir l'effet statistique de chaque marqueur génétique. L'ensemble des effets des marqueurs est combiné dans les équations de prévision qui donneront alors la valeur génétique des individus à sélectionner à partir de leur génotype et sans mesurer leur performance (Goddard et Hayes 2009). De plus, la sélection génomique affranchit la sélection des individus de la mesure de performance, celle-ci n'étant plus mesurée que dans la population de référence. Il est donc possible d'intégrer dans les schémas de sélection des caractères tels que la résistance aux SGI, leurs coûts de mesure étant trop élevés et/ou leurs impacts sur la santé étant non négligeables. A ce titre, la sélection pour la résistance aux maladies bénéficiera probablement de ces avancées. La limite de la sélection génomique sur des caractères tels que la résistance aux SGI est la taille de la population de référence : on estime qu'au moins 1000 individus sont nécessaires pour obtenir une valeur génétique estimée de précision suffi- sante (Goddard et Hayes 2009), ce qui implique un investissement de base considérable en phénotypage et génotypage. De plus, cet investissement doit être renouvelé régulièrement de manière à limiter l'impact des recombinaisons entre les gènes ciblés et les marqueurs observés et sélectionnés. En effet, les équations de prévision estimées à un temps $\mathrm{t}$ dans une population de référence sont susceptibles d'être moins précises au bout de plusieurs générations sans phénotypage. Cette perte de précision reste à estimer pour apprécier le coût d'une telle sélection génomique sur la résistance aux SGI chez les petits ruminants.

\section{Conclusion}

L'amélioration génétique apparaît être une voie prometteuse pour rétablir un équilibre entre les petits ruminants et leurs parasites gastro-intestinaux. Il faut avant tout capitaliser sur des génotypes adaptés au milieu c'est-à-dire à la pression parasitaire locale, notamment en zone tropicale. Leur résistance peut ensuite être augmentée efficacement par sélection conjointement à leurs performances de production. Ces nombreuses connaissances militent donc pour la prise en compte de ce caractère d'adaptation dans les schémas de sélection. L'application est encore limitée à quelques cas comme en Australie pour les ovins. C'est également l'option choisie par les éleveurs caprins guadeloupéens. Cependant, pour gagner en efficacité et en durabilité, la résistance génétique aux SGI doit être combinée dans une lutte intégrée à d'autres voies de maîtrise du parasitisme (gestion du pâturage, complémentation alimentaire, traitements ciblés...).

\section{Références}

Alba-Hurtado F., Romero-Escobedo E., Muñoz-Guzmán M.A., Torres-Hernández G., Becerril-Pérez C.M., 2010. Comparison of parasitological and productive traits of Criollo lambs native to the central Mexican Plateau and Suffolk lambs experimentally infection with Haemonchus contortus. Vet. Parasitol., 172, 277-282.

Albers G.A.A., Gray G.D., Piper L.R., Barker J.S.F., Le Jambre L.F., Barger I.A., 1987. The genetics of resistance and resilience to Haemonchus contortus infection in young Merino sheep. Int. J. Parasitol., 17, 1355-1363.

Alexandre G., Mandonnet N., 2005. Goat meat production in harsh environments. Small Rum. Res., 60, 53-66.

Amarante A.F.T., Craig T.M., Ramsey W.S., El-Sayed N.M., Desouki A.Y., Bazer F.W., 1999. Comparison of naturally acquired parasite burdens among Florida Native,
Rambouillet and crossbreed ewes. Vet. Parasitol., 85, 61-69.

Amarante A.F.T., Bricarello P.A., Rocha R.A., Gennari S.M., 2004. Resistance of Santa Ines, Suffolk and Ile de France sheep to naturally acquired gastrointestinal nematode infections. Vet. Parasitol., 120, 91-106.

Amarante A.F.T., Susin I., Rocha R.A., Silva M.B., Mendes C.Q., Pires A.V., 2009. Resistance of Santa Ines and crossbred ewes to naturally acquired gastrointestinal nematode infections. Vet. Parasitol., 165, 273-280.

Andronicos N., Hunt P., Windon R., 2010 Expression of genes in gastrointestinal and lymphatic tissues during parasite infection in sheep genetically resistant or susceptible to Trichostrongylus colubriformis and Haemonchus contortus. Int. J. Parasitol., 40, 417-429.
Aumont G., Gruner L., Hostache G., 2003. Comparison of the resistance to sympatric and allopatric isolated of Haemonchus contortus of Black Belly sheep in Guadeloupe (FWI) and of INRA 401 sheep in France. Vet. Parasitol., 116, 139-150.

Ayalew W., Rischkowsky B., King J.M., Bruns E., 2003. Crossbreds did not generate more net benefits than indigenous goats in Ethiopian smallholdings. Agric. Sys., 76, 1137-1156.

Baker R.L., 1997. Résistance génétique des petits ruminants aux helminthes en Afrique. INRA Prod. Anim., 10, 99-110.

Baker R.L., Gray G.D., 2003. Appropriate breeds and breeding schemes for sheep and goats in the tropics. Sani R.A., Gray G.D., Baker R.L. (Eds.) Worm Control for Small Ruminants in Tropical Asia, Monograph 113. Australian Centre for International Agricultural Research (ACIAR), 63-95. 
Baker R.L., Mwamachi D.M., Audho J.O., Thorpe W., 1994. Genetic resistance to gastrointestinal nematode parasites in red Maasai sheep in Kenya. In: session 38 Eds Smith C, Gavora J.S et al, 5th WCGALP Guelph, Canada, 20, 277-280.

Baker R.L., Mwamachi D.M., Audho J.O., Aduda E.O., Thorpe W., 1998. Resistance of Galla and Small East African goats in the subhumid tropics to gastrointestinal nematode infections and the peri-parturient rise in faecal egg counts. Vet. Parasitol., 79, 53-64.

Baker R.L., Audho J.O., Aduda E.O., Thorpe W., 2001. Genetic resistance to gastrointestinal nematode parasites in Galla and Small East African goats in the sub-humid tropics. Anim. Sci., 73, 61-70.

Baker R.L., Nagda S., Rodrigez-Zas S.L., Southey B.R., Audho J.O., Aduda E.O., Thorpe W., 2003. Resistance and resilience to gastrointestinal nematode parasites and relationships with productivity of Red Maasai, Dorper and Red Maasai x Dorper crossbred lambs in the sub-humid tropics. Anim. Sci., 76, 119136.

Bambou J.C., Gonzalez-Garcia E., de la Chevrotière C., Arquet R., Vachiery N., Mandonnet N., 2009. Peripheral immune response in resistant and susceptible Creole kids experimentally infected with Haemonchus contortus. Small Rum. Res., 82, 34-39.

Beh K.J., Hulme D.J., Callaghan M.J., Leish Z., Lenane I., Windon R.G., Maddox J.F., 2002. A genome scan for quantitative trait loci affecting resistance to Trichostrongylus colubriformis in sheep. Anim. Genet., 33, 97-106.

Beilharz R.G., Luxford B.G., Wilkinson J.L., 1993. Quantitative genetics and evolution: is our understanding of genetics sufficient to explain evolution? J. Anim. Breed. Genet. $110,161-170$.

Benavides M.V., Weimer T.A., Borba M.F.S., Berne M.E.A., Sacco A.M.S., 2002. Association between microsatellite markers of sheep chromosome 5 and faecal egg counts. Small Rum. Res., 46, 97-105.

Beraldi D., McRae A.F., Gratten J., Pilkington J.G., Slate J., Visscher P.M., Pemberton J.M., 2007. Quantitative Trait Loci (QTL) mapping of resistance to strongyles and coccidia in the free-living Soay sheep (Ovis aries). Int. J. Parasitol., 37, 121-129.

Bishop S.C., 2010. How to account for adaptation in breeding schemes for ruminant production under tropics: focus on resistance to strongyles in small ruminants? Advances in Animal Biosciences, 2010, 1(2) 384 (doi: 10.1017/S2040470010000129)

Bishop S.C., Morris C.A., 2007. Genetics of disease resistance in sheep and goats. Small Rum. Res., 70, 48-59.

Bishop S.C., Stear M.J., 2001. Inheritance of, and factors affecting, egg counts during early lactation in Scottish Blackface ewes facing mixed, natural nematode infections. Anim. Sci., 73, 389-395.

Bishop S.C., Jackson F., Coop R.L., Stear M.J. 2004. Genetic parameters for resistance to nematode infections in Texel lambs and their utility in breeding programmes. Anim. Sci., 78, 185-194.

Bolormaa S., Olayemi M., van der Werf J.H.J., Baillie N., Le Jambre F., Ruvinsky A., Walkden-Brown S.S., 2010a. Estimates of genetic and phenotypic parameters for production, haematological and gastrointestinal nema- tode-associated traits in Australian Angora goats. Anim. Prod. Sci., 50, 25-36.

Bolormaa S., van der Werf J.H.J., WalkdenBrown S.W., Marshall K., Ruvinsky A., 2010b. A quantitative trait locus for faecal worm egg and blood eosinophil counts on chromosome 23 in Australian goats. J. Anim. Breed. Genet. 127, 207-214.

Bouix J., Krupinski J., Rzepecki R., Nowosad B., Skrzyzala I., Roborzynski M. Fudalewicz-Niemczyk W., Skalska M. Malczewski A., Gruner L., 1998. Genetic resistance to gastrointestinal nematode parasites in Polish long-wool sheep. Int. J Parasitol., 28, 1797-1804.

Bricarello P.A., Gennari S.M., OliveiraSequeira T.C.G., Vaz C.M.S.L., Gonçalves de Gonçalves I., Echevarria F.A.M., 2004. Worm burden and immunological responses in Corriedale and Crioula Lanada sheep following natural infection with Haemonchus contortus. Small Rum. Res., 51, 75-83.

Buitkamp J., Filmether P., Stear M.J., Epplen J.T., 1996. Class I and class II majo histocompatibility complex alleles are associated with faecal egg counts following natural, predominantly Ostertagia circumcincta infection. Parasitol. Res., 82, 693-696.

Burke J.M., Miller J.E., 2002. Relative resistance of Dorper crossbred ewes to gastrointestinal nematode infection compared with St. Croix and Katahdin ewes in southeastern United States. Vet. Parasitol., 109, 265-275.

Burke J.M., Miller J.E., 2004. Relative resistance to gastrointestinal nematode parasites in Dorper, Katahdin, and St. Croix lambs under conditions encountered in the southeastern region of the United States. Small Rum. Res., 54, 43-51.

Carta, A., Casu S., Salaris S., 2009. Current state of genetic improvement in dairy sheep. J. Dairy Sci., 92, 5814-5833.

Charon K.M., Moskwa B., Rutkowski R., Gruszczynska J., Swiderek W., 2002 Microsatellite polymorphism in DRB 1 gene (MHC class II) and its relation to nematode faecal egg count in Polish Heath Sheep. J. Anim. Feed Sci., 11, 47-58.

Chauhan K.K., Rout P.K., Singh P.K Mandal A., Singh S.K., Roy R., 2003a. Genetic resistance of Barbari and Jamunapari kids to natural infection with gastrointestinal nematode. Trop. Anim. Health Prod., 35, 397-408.

Chauhan K.K., Rout P.K., Singh P.K., Mandal A., Singh H.N., Roy R., Singh S.K., 2003b. Susceptibility to natural gastro-intestinal nematode infection in different physiological stages in Jamunapari and Barbari goats in the semi-arid tropics. Small Rum. Res., 50, 219-223.

Chiejina S.N., Behnke J.M., Musongsong G.A., Nnadi P.A., Ngongeh L.A., 2010 Resistance and resilience of West African Dwarf goats of the Nigerian savanna zone exposed to experimental escalating primary and challenge infection with Haemonchus contortus. Vet. Parasitol., 171, 81-90.

Clarke R.A., Burn A.L., Lenane I., Windon R.G., Beh K.J., 2001. Molecular analysis an nematode resistance association of a polymorphism at the 5 ' end of the sheep IgE gene. Vet. Immunol. Immunopathol., 79, 15-29.

Colditz I., 2002. Effects of the immune system on metabolism: implications for production and disease resistance in livestock. Livest. Prod. Sci., 75, 257-268
Coltman D.W., Wilson K., Pilkington J.G., Stear M.J., Pemberton J.M., 2001. A microsatellite polymorphism in the gamma interferon gene is associated with resistance to gastrointestinal nematodes in a naturally-parasitized population of Soay sheep. Parasitologie, $122,571-582$

Costa C.A.F., Vieira L. da S., Berne M.E.A. Silva M.U.D., Guidoni A.L., Figueiredo E.A.P., 2000. Variability of resistance in goats infected with Haemonchus contortus in Brazil. Vet. Parasitol., 88, 153-158.

Crawford A.M., Paterson K.A., Dodds K.G., Diez Tascon C., Williamson P.A., Thomson M.R., Bisset S.A., Beattie A.E., Greer G.J., Green R.S., Wheeler R., Shaw R.J., Knowler K., McEwan J.C., 2006. Discovery of quantitative trait loci for resistance to parasitic nematode infection in sheep: I. Analysis of outcross pedigrees. BMC Genomics, 7, 178-187.

Davies G., Stear M.J., Bishop S.C., 2005 Genetic relationships between indicator traits and nematode parasite infection levels in 6month-old lambs. Anim. Sci., 80, 143-150.

Davies G., Stear M.J., Benothman M., Abuagob O., Kerr A., Mitchell S., Bishop S.C., 2006. Quantitative trait loci associated with parasitic infection in Scottish blackface sheep. Heredity, 96, 252-258.

Davis G.P., 1993. Genetic parameters for tropical beef cattle in northern Australia: a review. Aust. J. Agric. Res., 44, 179-198.

Diez-Tascon C., MacDonald P.A, Dodds K.G., McEwan J.C., Crawford, A.M., 2002. A screen of chromosome 1 for QTL affecting nematode resistance in an ovine outcross population. $7^{\text {th }}$ World Congress on Genetics Applied to Livestock Productions, Montpellier, France. Session 13, 13-37.

Diez-Tascón C., Keane O.M., Wilson T. Zadissa A., Hyndman D.L., Baird D.B. McEwan J.C., Crawford A.M., 2005. Microarray analysis of selection lines from outbred populations to identify genes involved with nematode parasite resistance in sheep. Physiol. Genomics, 21, 59-69.

Doeschl-Wilson A.B., Vagenas D., Kyriazakis I., Bishop S.C., 2008. Exploring the assumptions underlying genetic variation in host nematode resistance. Genet. Sel. Evol., 40, 241-264.

Dominik S., 2005. Quantitative trait loci for internal nematode resistance in sheep: review. Genet. Sel. Evol., 37, 83-96.

Emik L.O., 1949. The effects of environmental and hereditary factors on trichostrongylid worm infestation in sheep. J. Anim. Sci., 8, 73-80.

Finch V.A., 1986. Body temperature in beef cattle: its control and relevance to production in the tropics. J. Anim. Sci., 62, 531-542.

Gauly M., Erhardt G., 2001. Genetic resistance to gastrointestinal nematode parasites in Rhön sheep following natural infection. Vet. Parasitol., 102, 253-259.

Gauly M., Kraus M., Vervelde L., van Leeuwen M.A., Erhardt G., 2002. Estimating genetic differences in natural resistance in Rhön and Merinoland sheep following experimental Haemonchus contortus infection.Vet Parasitol., 106, 55-67.

Gicheha M.G., Kosgey I.S., Bebe B.O., Kahi A.K., 2005. Economic values for resistance to gastrointestinal helminths in meat sheep in Kenya. J. Anim. Breed. Genet., 122, 165-171. 
Gicheha M.G., Kosgey I.S., Bebe B.O., Kahi A.K., 2007. Efficiency of alternative schemes breeding for resistance to gastrointestinal helminths in meat sheep. Small Rum. Res., 69, 167-179.

Goddard M.E., Hayes B.J., 2009. Mapping genes for complex traits in domestic animals and their use in breeding programmes. Nat. Rev. Genet., 10, 381-391.

González J.F., Hernández Á., Molina J.M., Fernández A., Raadsma H.W., Meeusen E.N.T., Piedrafita D., 2008. Comparative experimental Haemonchus contortus infection of two sheep breeds native to the Canary Islands. Vet. Parasitol., 153, 374-378.

Good B., Hanrahan J.P., Crowley B.A., Mulcahy G., 2006. Texel sheep are more resistant to natural nematode challenge than Suffolk sheep based on faecal egg count and nematode burden. Vet. Parasitol., 136, 317-327.

Goossens G., Osaer S., Ndao M., Van Winghem J., Geerts S., 1999. The susceptibility of Djallonké and Djallonké-Sahelian crossbred sheep to Trypanosoma congolense and helminth infection under different diet levels. Vet. Parasitol., 85, 25-41.

Gregory P.W., 1937. The possibility of establishing within breeds lines of sheep that are genetically resistant to stomach worms. Am. Soc. Anim. Prod., 316-324.

Gruner L., Aumont G., Getachew T., Brunel J.C., Pery C., Cognié Y., Guérin Y., 2003. Experimental infection of Black Belly and INRA 401 straight and crossbred sheep with trichostrongyle nematode parasites. Vet. Parasitol., 116, 239-249.

Gruner L., Bouix J., Brunel J.C., 2004. High genetic correlation between resistance to Haemonchus contortus and to Trichostrongylus colubriformis in INRA 401 sheep. Vet. Parasitol., 119, 51-58.

Gunia M., Mandonnet N., Arquet R., de la Chevrotière C., Naves M., Mahieu M., Alexandre G., 2010. Production systems of Creole goat and their implications for a breeding programme. Animal, 4, 2099-2105.

Gunia M., Phocas F., Arquet R., Alexandre G., Mandonnet N., 2011. Genetic parameters for weight, reproduction and parasitism resistance traits in Creole goat. J. Anim. Sci. (sous presse) doi :10.2527/jas.2011-3872.

Gutiérrez-Gil B., Pérez J., Alvarez L., Martínez-Valladares M., de la Fuente L.F. Bayón Y., Meana A., San Primitivo F., RojoVázquez F.A., Arranz J.J., 2009. Quantitative trait loci for resistance to trichostrongylid infection in Spanish Churra sheep. Genet. Sel. Evol., 28, 41-46.

Haile A., Tembely S., Anindo D.O., MukasaMugerwa E., Rege J.E.O., Yami A., Baker R.L., 2002. Effects of breed and dietary protein supplementation on the responses to gastrointestinal nematode infections in Ethiopian sheep. Small Rum. Res., 44, 247-261.

Herbert J., Isham V., 2000. Stochastic hostparasite interaction models. J. Math. Biol. 40, 343-371.

Hielscher A., Brandt H., Erhardt G., Gauly M., 2006. Heterosis analysis of Haemonchus contortus resistance and production traits in Rhoen sheep, Merino Land sheep and crossbred lambs. Vet. Parasitol., 141, 279-284.

Hofmann R. R., 1989. Evolutionary steps of ecophysiological adaptation and diversification of ruminants: a comparative view of their digestive system. Oecologia, 78, 443-457.

Hoste H., Leveque H., Dorchies Ph., 2001. Comparison of nematode infections of the gastrointestinal tract in Angora and dairy goats in a rangeland environment: relations with the feeding behaviour. Vet. Parasitol., 101, 127135 .

Janssen M., Weimann C., Gauly M., Erhardh G., 2002. Associations between infections with Haemonchus contortus and genetic markers on ovine chromosome $20.7^{\text {th }}$ World Congress on Genetics Applied To Livestock Productions, Montpellier, France. Session 13, 13-11.

Keane O.M., Dodds K.G., Crawford A.M., McEwan J.C., 2007. Transcriptional profiling of Ovies aries identifies Ovar-DQA1 allele frequency differences between nematode-resistant and susceptible selection lines. Physiol. Genomics, 30, 253-261.

Kemper K. E., Elwin R. L., Bishop S. C., Goddard M. E., Woolaston R. R., 2009. Haemonchus contortus and Trichostrongylus colubriformis did not adapt to long-term exposure to sheep that were genetically resistant or susceptible to nematode infections. Int. J. Parasitol., 39, 607-614.

Khusro M., Van der Werf J.H.J., Brown D.J., Ball A., 2004. Across flock (co)variance components for faecal worm egg count, live weight, and fleece traits for Australian merinos. Livest. Prod. Sci., 91, 35-43.

Li Y., Miller J.E., Franke D.E., 2001. Epidemiological observations and heterosis analysis of gastrointestinal nematode parasitism in Suffolk, Gulf Coast Native, and crossbred lambs. Vet. Parasitol., 98, 273-283.

MacKinnon K.M., Burton J.L., Zajac A.M Notter D.R., 2009. Microarray analysis reveals difference in gene expression profiles of hair and wool sheep infected with Haemonchus contortus. Vet. Immunol. Immunopathol., 130, 210-220.

MacKinnon K.M., Zajac A.M., Kooyman F.N.J., Notter D.R., 2010. Differences in immune parameters are associated with resistance to Haemonchus contortus in Caribbean hair sheep. Parasite Immunol., 32, 484-493.

Mahieu M., Arquet R., Kandassamy T., Mandonnet N., Hoste H., 2007. Evaluation of targeted drenching using Famacha method in Creole goat: reduction of anthelmintic use, and effects on kid production and pasture contamination. Vet. Parasitol., 146, 135-147.

Mahieu M., Arquet R., Fleury J., Coppry O., Marie-Magdeleine C., Boval M., Archimède H., Alexandre G., Bambou J.C., Mandonnet N., 2009. Contrôle intégré du parasitisme gastro-intestinal des petits ruminants au pâturage en zone tropicale humide. In : Santé - Sécurité des aliments. Institut de l'Élevage (Eds.). 16 eme Rencontres Recherches Ruminants, Paris, France, 265-268.

Makun H.J., Ajanusi J.O., Lakpini C.A.M., Ehoche O.W., Rekwot P.I., 2008. Response of Red Sokoto and Sahelian goats to trickle Haemonchus contortus infection. J. Biol. Sci., 8, 753-759.

Mandal A., Sharma D.K., 2008. Inheritance of faecal nematode egg count in Barbari goats following natural Haemonchus contortus infection. Vet. Parasitol., 155, 89-94.

Mandonnet N., Aumont G., Fleury J., Arquet R., Varo H., Gruner L., Bouix J., Vu-Tien Khang J., 2001. Assessment of genetic variability of resistance to gastrointestinal nematode parasites in Creole goats in the humid tropics. J. Anim. Sci., 79, 1706-1712.

Mandonnet N., Ducrocq V., Arquet R., Aumont G., 2003. Mortality of Creole kids during infection with gastrointestinal strongyles: A survival analysis. J. Anim. Sci., 81, 2401-2408.

Mandonnet N., Menendez-Buxadera A. Arquet R., Mahieu M., Bachand M., Aumont G., 2006. Genetic variability in resistance to gastro-intestinal strongyles during early lactation in Creole goats. Anim. Sci., 82, 283-287.

Marshall K., Maddox J.F., Lee S.H., Zhang Y., Kahn L., Graser H.U., Gondro C., WalkdenBrown S.W., van der Werf J.H.J., 2009. Genetic mapping of quantitative trait loci for resistance to Haemonchus contortus in sheep. Anim. Genet., 40, 262-272.

Matika O., Nyoni S., van Wyk J.B., Erasmus G.J., Baker R.L., 2003. Resistance of Sabi and Dorper ewes to gastro-intestinal nematode infections in an African semi-arid environment. Small Rum. Res., 47, 95-102.

McEwan J.C., Mason P., Baker R.L., Clarke J.N., Hickey S.M., Turner K., 1992. Effect of selection for productive traits on internal parasite resistance in sheep. Proc. N.Z. Soc. Anim. Prod., 52, 53-56.

McManus C., Louvandini H., Paiva S.R., de Oliveira A.A., Azevedo H.C., de Melo C.B., 2009. Genetic factors of sheep affecting gastrointestinal parasite infections in the Distrito Federal, Brazil. Vet. Parasitol., 166, 308-313.

Menendez-Buxadera A., Mandonnet N., 2006. The importance of the genotype*environment interaction for selection and breeding programmes in tropical conditions, CAB Reviews: Perspectives in Agriculture, Veterinary Science, Nutrition and Natural Resources, 26, 14 p.

Mignon-Grasteau S., Boissy A., Bouix J., Faure J.M., Fisher A.D., Hinch G.N., Jensen P., Le Neindre P., Mormède P., Prunet P., Vandeputte M., Beaumont C., 2005. Genetics of adaptation and domestication in livestock. Livest. Anim. Sci., 93, 3-14.

Miller J.E., Bahirathan M., Lemarie S.L., Hembry F.G., Kearney M.T., Barras S.R., 1998. Epidemiology of gastrointestinal nematode parasitism in Suffolk and Gulf Coast Native sheep with special emphasis on relative susceptibility to Haemonchus contortus infection. Vet. Parasitol., 74, 55-74.

Miller J.E., Bishop S.C., Cockett N.E., McGraw R.A., 2006. Segregation of natural and experimental gastrointestinal nematode infection in $\mathrm{F}_{2}$ progeny of susceptible Suffolk and resistant Gulf Coast Native sheep and its usefulness in assessment of genetic variation. Vet. Parasitol., 140, 83-89.

Mirkena T., Duguma G., Haile A., Tibbo M., Okeyo A.M., Wurzinger M., Sölkner J., 2010. Genetics of adaptation in domestic farm animals: A review. Livest. Sci., 132, 1-12.

Moreno C.R., Gruner L., Scala A., Mura L., Schibler L., Amigues Y., Sechi T., Jacquiet P., François D., Sechi S., Roig A., Casu S., Barillet F., Brunel J.C., Bouix J., Carta A., Rupp R., 2006. QTL for resistance to internal parasites in two designs based on natural and experimental conditions of infection. $8^{\text {th }}$ World Congress on Genetics Applied to Livestock Productions, Belo Horizonte, Brazil, Session 15, 15-05. 
Morris C.A., Vlassoff A., Bisset S.A., Baker R.L., West C.J., Hurford A.P., 1997a. Responses of Romney sheep to selection for resistance or susceptibility to nematode infection. Anim. Sci., 64, 319-329.

Morris C.A., Wheeler M., Hosking B.C., Watson T.G., Hurford A.P., Foote B.J., Foote J.F., 1997b. Genetic parameters for milk yield and faecal nematode egg count in Saanen does. N.Z. J. Agric. Res., 40, 523-528.

Morris C.A., Bisset S.A., Vlassoff A., West C.J, Wheeler M., 1998. Faecal nematode egg counts in lactating ewes from Romney flocks selectively bred for divergence in lamb faecal egg count. Anim. Sci., 67, 283-288.

Morris C.A. Bisset S.A., Vlassoff A., West C.J., Wheeler M., 2004. Genetic parameters for Nematodirus spp. egg counts in Romney lambs in New Zealand. Anim. Sci., 79, 33-39.

Morris C.A., Wheeler M., Watson T.G., Hosking B.C., Leathwick D.M., 2005. Direct and correlated responses to selection for high or low faecal nematode egg count in Perendale sheep. N.Z. J. Agric. Res., 48, 1-10.

Mugambi J.M., Wanyangu S.W., Bain R.K., 1996., Response of Dorper and red Maasai lambs to trickle Haemonchus contortus infections. Res. Vet. Sci., 61, 218-221.

Mugambi J.M., Bain R.K., Wanyangu S.W., Ihiga M.A., Duncan J.L., Murray M., Stear M.J., 1997. Resistance of four sheep breeds to natural and subsequent artificial Haemonchus contortus infection. Vet. Parasitol., 69, 265-273.

Neurath M. F., Finotto S., Glimcher L. H., 2002. The role of Th $1 /$ Th 2 polarization in mucosal immunity. Nature Medicine, 8, 567-573.

Nimbkar C., Ghalsasi P.M., Walkden-Brown S.W., Khan L.P., 2002. Breeding program for the genetic improvement of Deccani sheep of Maharashtra, India. In: Developing sustainable breeding strategies in medium-to low-input systems. $7^{\text {th }}$ World Congress on Genetics Applied to Livestock Productions, Montpellier, France, Session 25, 25-11.

Nimbkar C., Ghalsasi P.M., Swan A.A. Walkden-Brown S.W., Khan L.P., 2003. Evaluation of growth rates and resistance to nematodes of Deccani and Bannur lambs and their crosses with Garole. Anim. Sci., 76, 503515 .

Over H.J., Jansen J., Olm P.W.V., 1992. Distribution and impact of helminth diseases of livestock in developing countries. FAO Anim. Prod. Health Pap., 96, 221.

Paterson S., Wilson K., Pemberton J.M., 1998. Major histocompatibility complex variation associated with juvenile survival and parasite resistance in a large unmanaged ungulate population (Ovis aries L.). Proc. Natl. Acad. Sci. U.S.A., 95, 3714-3719.

Poivey J.P., 1987. Development of breeding methods in the tropics with limited availability of in-the-field recording systems. World Review of Animal Production, 23, 83-92.

Pollot G.E., Karlsson L.J.E., Eady S., Greeff J.C., 2004. Genetic parameters for indicators of host resistance to parasites from weaning to hogget age in Merino sheep. J. Anim. Sci., 82, 2852-2864.

Pralomkarn W., Pandey V.S., Ngampongsai W., Choldumrongkul S., Saithanoo S., Rattaanachon L., Verhulst A., 1997. Genetic resistance of three genotypes of goats to exper- imental infection with Haemonchus contortus. Vet. Parasitol., 68, 79-90.

Prince L.L.L., Gowane G.R., Swarnkar C.P., Singh D., Arora A.L., 2010. Estimated of genetic parameters for faecal egg count of Haemonchus contortus infection and relationship with growth traits in Avikalin sheep. Trop. Anim. Health Prod., 42, 785-791.

Rahman W.A., Collins G.H., 1990. The establishment and development of Haemonchus contortus in goats. Vet. Parasitol. $35,189-193$.

Rege J.E.O., Tembely S., Mukasa-Mugerwa E., Sovani A., Anindo D., Lahlou-Kassi A. Nagda S., Baker R.L., 2002. Effect of breed and season on production and response to infection with gastro-intestinal nematode parasites in sheep in the highlands of Ethiopia Livest. Prod. Sci., 78, 159-174.

Romjali E., Dorny P., Batubara A., Pandey V.S., Gatenby R.M., 1997. Peri-parturient rise in faecal strongyle egg counts of different genotypes of sheep in North Sumatra Indonesia. Vet. Parasitol., 68, 191-196.

Rowe A., Gondro C., Emery D., Sangster N., 2009. Sequential microarray to identify timing of molecular responses to Haemonchus contor tus infection in sheep. Vet. Parasitol., 161, 7687.

Saddiqi H.A., Iqbal Z., Khan M.N., Muhhamad G., 2010a. Comparative resistance of sheep breeds to Haemonchus contortus in a natural pasture infection. Int. J. Agric. Biol., 12, 739-743.

Saddiqi H.A., Iqbal Z., Khan M.N., Sarwar M., Muhhamad G., Yaseen M., Jabbar A., 2010b. Evaluation of three Pakistani sheep breeds for their natural resistance to artificial infection of Haemonchus contortus. Vet. Parasitol., 168, 141-145.

Safari E., Fogarty N.M., Gilmour A.R. 2005. A review of genetic parameter estimates for wool, growth, meat and reproduction traits in sheep. Livest. Prod. Sci., 92, 271-289.

Sangster N.C., 1999. Anthelmintic resistance: past, present and future, Int. J. Parasitol., 29, 115-124.

Saulai M., Cabaret J., Hostache G., Mandonnet N., Aumont G., 2001. Life-trait evolution of a parasite strongyle nematode in response to host resistance: an experimenta approach using Haemonchus contortus in black belly lambs. Genet. Sel. Evol., 33, 25-44.

Sayers G., Good B., Hanrahan J.P., Ryan M., Angles M., Sweeney T., 2005a. Major Histocompatibility complex DRB1 gene: its role in nematode resistance in Suffolk and Texel sheep breeds. Parasitology 131, 403-409.

Sayers G., Good B., Hanrahan J.P., Ryan M. Sweeney T., 2005b. Intron 1 of the interferon? gene: Its role in nematode resistance in Suffolk and Texel sheep breeds. Res. Vet. Sci., 79, 191196.

Schwaiger F.W., Gostomski D., Stear M.J. Duncan J.L., McKellar Q.A., Epplen J.T. Buitkamp J., 1995. An ovine major histocompatibility complex Drb1 allele is associated with low fecal egg counts following natural, predominantly Ostertagia circumcincta infection. Int. J. Parasitol., 25, 815-822.

Sourdioux M., Lagarrigue S., Douaire M., 1997. Analyse génétique d'un caractère quantitatif. Inra Prod. Anim., 10, 251-258.
Sréter T., Kassai T., Takacs E., 1994. The heritability and specificity of responsiveness to infection with Haemonchus contortus in sheep. Int. J. Parasitol., 24, 871-876.

Tariq K.A., Chishti M.Z., Ahmad F., Shaw A.S., 2008. Epidemiology of gastrointestinal nematodes of sheep managed under traditional husbandry system in Kashmir valley. Vet. Parasitol., 158, 138-143.

Tembely S., Lahlou-Kassi A., Rege J.E.O., Mukasa-Mugerwa E., Anindo D., Sovani S., Baker R.L., 1998. Breed and season effects on the peri-parturient rise in nematode egg output in indigenous ewes in a cool tropical environment. Vet. Parasitol., 77, 123-132.

Terefe G., Lacroux C., Andreoletti O. Grisez C., Prevot F., Bergeaud J.P., Penicaud J., Rouillon V., Gruner L., Brunel J.C., François D., Bouix J., Dorchies P., Jacquiet P., 2007. Immune response to Haemonchus contortus infection in susceptible (INRA 401) and resistant (Barbados Black Belly) breeds of lambs. Parasite Immunol., 29, 415-424.

Thamsborg S.M., Jorgensen R.J., Waller P.J., Nansen P., 1996. The influence of stocking rate on gastrointestinal nematode infections of sheep over a 2-year grazing period. Vet. Parasitol., 67, 207-224.

Vagenas D., Jackson F., Russel A.J.F., Merchant M., Wright I.A., Bishop S.C., 2002. Genetic control of resistance to gastro-intestinal parasites in crossbred cashmere-producing goats: responses to selection, genetic parameters and relationships with production traits. Anim. Sci., 74, 199-208

Vanimisetti H.B., Andrew S.L., Zajac A.M., Notter D.R., 2004. Inheritance of fecal egg count and packed cell volume and their relationship with production traits in sheep infected with Haemonchus contortus. J. Anim. Sci. $82,1602-1611$

Vercoe J.E., Frisch J.E., 1992. Genotype (breed) and environment interaction with particular reference to cattle in the tropics. AsianAustr. J. Anim. Sci., 5, 401-409.

Waller P.J., 1997. Nematode parasite control of livestock in the tropics/subtropics: the need for novel approaches. Int. J. Parasitol., 27, 1193-1201.

Wanyangu S.W., Mugambi J.M., Bain R.K., Duncan J.L., Murray M., Stear M.J., 1997. Response to artificial and subsequent natural infection with Haemonchus contortus in Red Maasai and Dorper ewes. Vet. Parasitol., 69, 275-282

Watson T.G., Hosking B.C., Hurford A.P. Mather B.C., 1992. Developments in breeding Perendale sheep for resistance or susceptibility to internal nematode parasites. Proc. N.Z. Soc. Anim. Prod., 52, 61-64

Watson T.G., Baker R.L., Harvey T.G. 1986. Genetic variation in resistance or tolerance to internal nematode parasites in strains of sheep at Rotomahana. Proc. N.Z. Soc. Anim. Prod., 46, 23-26.

Wolf B.T., Howells K., Nakielny C., Haresign W., Lewis R.M., Davies O., Davies M.H., 2008. Genetic parameters for strongyle and Nematodirus faecal egg counts in lambs and their relationships with performance traits. Livest. Sci., 113, 209-217.

Woolaston R.R., Eady S.J., 1995. Australian research on genetic resistance to nematode parasites, In: Gray G.D., Woolaston R.R., Eaton B.T. (Eds.), Breeding for Resistance to 
Infectious Diseases of Small Ruminants, Austr. Centre Int. Agric. Res., Canberra, Australia, 53-75.

Woolaston R.R., Piper L.R., 1996. Selection of Merino sheep for resistance to Haemonchus contortus: genetic variation. Anim. Sci., 62, $451-460$
Woolaston R.R., Windon R.G., 2001. Selection of sheep for response to Trichostrongylus colubriformis larvae: genetic parameters. Anim. Sci., 73, 41-48.

Woolaston R.R., Windon R.G., Gray G.D., 1991. Genetic variation in resistance to internal parasites in Armidale experimental flocks. In: Breeding for disease resistance in sheep. Gray
G.D., Woolaston R.R. (Eds). Australian Wool Corporation Editions, Melbourne, Australie, 1-9.

Woolaston R.R., Singh R., Tabunakawai N., Le Jambre L.F., Banks D.J.P., Barger I.A., 1992. Genetic and environmental influences on worm egg counts of goats in the humid tropics. Proc. Austr. Assoc. Anim. Breed. Genet., 10, $147-150$.

\section{Résumé}

La durabilité des systèmes d'élevage tropicaux et tempérés extensifs est à rechercher dans l'équilibre entre le milieu et les productions, animales et végétales. Ainsi, il est judicieux de choisir les animaux pour leur adaptation aux contraintes du milieu, plutôt que de chercher à les en soustraire. Dans ce contexte, les strongyloses gastro-intestinales représentent une contrainte pathologique majeure des petits ruminants, particulièrement en zone tropicale humide. Depuis quelques années, la stratégie d'éradication des parasites a évolué vers une logique de manipulation des équilibres hôtes-parasites dans les systèmes pâturés par combinaison de diverses stratégies. La résistance génétique aux strongles gastro-intestinaux s'inscrit dans cette nouvelle démarche et y tient un rôle majeur. L'objectif de cette synthèse est de souligner les apports de la génétique et de la génomique à la compréhension et à l'exploitation de différences entre individus pour la résistance aux strongyloses gastro-intestinales. Les races locales se révèlent dans la plupart des cas les plus résistantes et les plus productives dans leur milieu. Que ce soit chez des races de milieu tropical ou tempéré, une variabilité génétique intra-race a pu être observée chez les ovins et les caprins. Des critères de résistance exploitables en sélection sont connus chez les ovins comme chez les caprins avec un contrôle génétique comparable. Enfin, la composante immunitaire (complexe majeur d'histocompatibilité notamment) de la résistance est clairement impliquée dans les mécanismes. Cependant, du fait de comportements alimentaires différents, les mécanismes sous jacents pourraient être plus simples et moins efficients chez les caprins. L'information génomique s'accumule mais reste encore difficilement valorisable par les professionnels.

\section{Abstract}

\section{Selection tools for the control of gastrointestinal parasitism in small ruminants}

The sustainability of tropical and extensive temperate farming systems is the key for a balance between the environment (soil, fauna and flora) and animal and plant productions. The restoration or preservation of such a trophic and ecological balance requires the implementation of innovative techniques. It is vain to avoid constraints in animal rearing and wiser to choose animals for their adaptations to these constraints. In this context, gastrointestinal strongyle infections are a major constraint in small ruminants, particularly in the humid tropics. In recent years, the strategy of pest eradication has evolved to a more logical manipulation of hostparasite equilibrium in grazed systems by implementation of various actions. The genetic resistance of small ruminants to gastrointestinal strongyle infections is part of this new approach and plays a major role. The objective of this review was to highlight the contributions of quantitative genetics and genomics to the understanding and exploitation of the differences between individuals for resistance to gastrointestinal strongyle infections. Local breeds are found, in most cases, to be the most resistant and more productive in their environment. Genetic variability exploitable in selection is known in sheep as in goats with a similar genetic control. However, the underlying biological mechanisms are more basic and less efficient in goats. The genomic information accumulates but remains difficult to exploit by professionals.

DE LA CHEVROTIÈRE C., MORENO C., JAQUIET P., MANDONNET N., 2011. La sélection génétique pour la maîtrise des strongyloses gastro-intestinales des petits ruminants. INRA Prod. Anim., 24, 221-234. 